\title{
Supporting students' conceptual development of light refraction by simulation-based open inquiry with dual-situated learning model
}

\author{
Niwat Srisawasdi · Siriporn Kroothkeaw
}

Received: 15 December 2013/Revised: 15 February 2014/Accepted: 5 March 2014/

Published online: 2 April 2014

(C) Beijing Normal University and Springer-Verlag Berlin Heidelberg 2014

\begin{abstract}
Although light is an everyday phenomenon that we constantly observe, a numerous researches have reported that students often displayed learning difficulties and hold unscientific understanding on physics concepts of light wave. To address the situation, inquiry-based learning process with a support of computer simulation has been proved its benefits on development of student's conceptual learning in science. This paper presents the effects of simulation-based open inquiry with dualsituated learning model on forty 11th grade students' conceptual understanding of light refraction phenomena and change of their conceptions through mixed research methodology. The concurrent mixed methods strategy of one-group pre-, post-, and retention-quasi-experimental design and phenomenological research design was used in this study. The result showed that the students' conceptual understanding scores for pre-, post-, and retention tests were significantly different and their understanding could be improved after participating with the learning program, which is consistent with a result regarding the quantity of conceptual change. The evidence also indicated that mechanism of conceptual change induced the students' progression of scientific conceptual understanding of light refraction. Moreover, the result revealed that the later scientific understanding obtained after the participation was preserved within the students' cognitive structure of conceptual knowledge. This finding suggests that the learning program of simulation-based open inquiry with dual-situated learning model could be used to support a more meaningful learning in science concepts through the process of conceptual change.
\end{abstract}

\footnotetext{
N. Srisawasdi $(\bowtie)$

Division of Science, Mathematics, and Technology Education, Faculty of Education, Khon Kaen University, Khon Kaen, Thailand

e-mail: niwsri@kku.ac.th

S. Kroothkeaw

Office of the Basic Education Commission, Kaennakhon Witthayalai School, Khon Kaen, Thailand e-mail: ksiriporn@gmail.com
} 
Keywords DSLM · Open inquiry · Computer simulation · Conceptual understanding $\cdot$ Conceptual change

\section{Introduction}

In general, scientific concepts of light refraction are basic and yet important contents in physics education. In the context of Thailand basic education, students need to gain this scientific concept properly in order to understand related and advanced physics concepts in the future, i.e., interference of light wave and spectrum of light. Without understanding the concept of light and its properties, students may not understand many scientific domains (Djanett et al. 2013). Unfortunately, researchers have reported that the Thai students hold alternative conceptions in science phenomena about refraction of light. A few examples are they confused about the meaning of light reflection and refraction; the direction of propagation of light; how light refraction occurs at an interface; and how to determine a position of image (Kaewkhong et al. 2010). These alternative conceptions arise because of their preexisting ideas and beliefs based on their everyday experience with the light (Galili and Hazan 2001). The theoretical parameters associated with light, such as its speed, wave length, pressure, and discrete nature, are all far removed from the range of perceptions of the human senses, and in the case of optics instruction which is heavily based on graphic symbolism and which is subject to interpretation, there is a need for careful consideration in physics teaching process. However, students find the subject of optics to be obscure and difficult, and teachers help is often inadequate (Galili and Hazan 2000, 2001) because of its complex and abstract relations. These learning difficulties can be significantly reduced by embedding essential process and content explanations within the classroom learning environment. Unfortunately, students face most of the light concepts in school learning in the context where teachers mostly use the traditional transmission model, and in this case, Thailand is no exception. The traditional transmission model of teaching is ineffective in physics concepts learning (Dykstra et al. 1992; Hake 1998) because the teaching process of delivery of new knowledge to students does not focus on detecting their pre-existing ideas and beliefs and correcting their alternative conceptions (Jaakkolaa and Nurmi 2008; Jaakkola et al. 2011). As such, new instructional strategies must be developed to assist in active construction and meaningful adaptation of their knowledge (de Jong and Van Joolingen 1998). In order to help student a meaningful understanding of the refraction of light phenomena, students' alternative conceptions, therefore, must be established and removed (Aydin et al. 2012). Therefore, a learning process in which students' alternative conceptions were changed, transformed, or reconstructed into the intended scientific conceptions was officially called for physics instruction in Thailand.

In recent years, to promote students' scientific understanding and conceptual change, several researchers have attempted to develop instructional materials and researches for teaching of physics concepts: for example, Newton's laws of motion (Atasoy and Akdeniz 2007; Macabebe et al. 2010; Saglam-Arslan and Devecioglu 
2010; Savinainen and Scott 2002; Spyrtou et al. 2009), optics (Djanett et al. 2013; Kaewkhong et al. 2010), sound wave (Gunhaart and Srisawasdi 2012), climate change (Lombardi et al. 2013), air pressure and buoyancy (She 2002), thermal expansion (She 2003), heat transfer (She 2004b), electricity and magnetism (Dega et al. 2013), and electricity (Chiu and Lin 2005; Jaakkolaa and Nurmi 2008; Jaakkolaa et al. 2011; Zacharia 2007). These researchers showed the importance of cognitive domain on learners' process of conceptual change toward better conceptual understanding. With the importance of studying pedagogical research and development in science learning, many instructional models were grounded on the theoretical aspects of conceptual change for several decades: for example, conceptual change model (Posner et al. 1982), generative learning model (Cosgrove and Osborne 1985), cooperative learning model (Tao and Gunstone 1999), dualsituated learning model (DSLM) (She 2002, 2003, 2004), model-based inquiry (Stewart et al. 2005); these models aim to introduce cognitive conflicts into learner's cognitive structure and then allow them to adjust the existing conceptual understanding into a balance through a process of conceptual change.

The DSLM (She 2002, 2003, 2004a, b; Lee and She 2010; Liao and She 2009; She and Liao 2010; Tseng et al. 2010; Yen et al. 2011) is one of the instructional models which considers students' alternative conceptions to be a very important consideration in process of learning. In the DSLM, the nature of science concepts was emphasized, together with students' ontological and epistemological beliefs of science concepts as its major theoretical constructs for conceptual change (Liao and She 2009; She and Liao 2010). As such, DSLM provides a unique direction to emphasize that the learning process of conceptual change should be situated in the nature of science concepts and students' beliefs of these science concepts. These features need to be combined into designing of learning events and instructing essential mental sets needed for revising their alternative conceptions and constructing more complete scientific conceptions (She 2002, 2004a, b; She and Liao 2010). Based on the DSLM, cognitive dissonance has been created with students' preconceptions while providing students with correct conceptions to challenge their previous mental set, and this cognitive process function student's change of conceptual understanding. As per previous studies, the DSLM has demonstrated its potential to facilitate students' conceptual change involving the various attributes of science concepts such as air pressure and buoyancy (She 2002), thermal expansion (She 2003), dissolution and diffusion (She 2004a), heat transfer (She 2004b), atoms (Liao and She 2009; She and Liao 2010), acid-base-salt (Tseng et al. 2010), combustion (Lee and She 2010), and chemical reaction (Yen et al. 2011). In addition, these successful concepts of teaching of science promote conceptual change involving both invisible as well as process attributes by means of laboratory (She 2002, 2003, 2004a, b) and web-based environment (Lee and She 2010; Liao and She 2009; She and Liao 2010; Tseng et al. 2010; Yen et al. 2011) as conceptual-change learning materials. Interestingly, no study has investigated other types of learning material (e.g., microcomputer-based laboratory, computer simulation and animation, mobile environment, and augmented reality) that may influence students' conceptual change associated to this model. 
Currently, with the rapid growth of computers and technologies in the practice and progression of science education community, contemporary technology-based approach to science learning offers computer simulation with ample opportunities for students' inquiry-related use (Vreman-de Olde et al. 2013). By placing emphasis on the learner as an active agent in the process of conceptual change, computer simulations can support inquiry learning practices (de Jong and van Joolingen 1998; Rutten et al. 2012). Simulation-based inquiry has been becoming a pedagogical approach for enhancing students' conceptual learning in school science. By interacting with this approach, students practice scientific method in the context of performing their own investigations, while at the same time develop knowledge of the concept modeled by the simulation (de Jong et al. 2013; Lazonder et al. 2010; Vreman-de Olde et al. 2013).

According to the learning problems in science (i.e., refraction of light) outlined in the previous section, Suits and Srisawasdi (2013) mentioned the affordabilities of instructional computer simulation, which could support perceptions of students to visualize scientific phenomena both macroscopic, microscopic, and symbolic levels of representation. The situational complex and abstract relations of science concepts could be graphically presented with the capability of computer simulation (Zacharia and Anderson 2003). In that environment, it provides students with a "cleaned-up," idealized version of the complex and messy real world, while still retaining a necessary level of theoretical authenticity (Hennessy et al. 2006). Simulation-based inquiry allows students to explore unobservable phenomena; link observable and unobservable phenomena; point out salient information; enable learners to conduct multiple experiments in a short duration of time; and provide results of lengthy investigations instantaneously (de Jong et al. 2013). These capabilities make simulation a promising tool for promoting conceptual change (Tao and Gunstone 1999; Zacharia and Anderson 2003; Blake and Scanlon 2007). Previous studies have shown that simulation-based inquiry learning environment, as an active agent in the process of conceptual change, effectively promotes better conceptual understanding in science for students (Hsu 2008; Jaakkola and Nurmi 2008; Jaakkola et al. 2011; Lazonder and Ehrenhard 2013; McElhaney and Linn 2011; Olympiou and Zacharia 2012; Olympiou et al. 2013; Renken and Nunez 2013; Suits and Srisawasdi 2013; Trundle and Bell 2010; Zacharias et al. 2008). However, radical change of misconceptions involves more complex mental activity, and needs appropriate instructional strategy which creates dissonance with students' preexisting knowledge and provides new mental sets for them to achieve a more scientific view of the concept, which can be either a revision of the old model or the construction of a newone (She 2004a, b). Further the interest of conceptual change research, it is challenge to extend the potential of simulation-based inquiry into DSLM to make conceptual change less difficult and speed up the process, particularly of radical conceptual change.

In educational practice, approach of simulation-based inquiry could be incorporated into the DSLM pedagogical platform for inducing cognitive mechanism of radical conceptual change. Common characteristics of computer simulation (e.g., 2D and 3D model-based visualization, macro-microscopic interplay, graphical dynamic generation by interactivity, scaffoldings and supports) are appropriate to 
use for supporting features of DSLM. She and Liao (2010) argued that the use of graphic and text illustrations, simulated 2D animations, 3D animations, and simulated experiments must create dissonance and provide students with new mental sets, and challenge their ontological and epistemological beliefs. Moreover, instructional activity of scientific inquiry could be used to provide students with opportunities to visualize what actually happens, motivate them to reconstruct the mental sets they lack, and revise alternative conceptions (She 2004b). As DSLM has been mentioned to be a better teaching model for conceptual change as well as in simulation-based inquiry, no study has investigated impacts of simulation-based inquiry with DSLM on students' conceptual development.

\section{Literature review}

Conceptual change and dual-situated learning model (DSLM)

In several past decades, researches have proposed a variety of theories regarding conceptual change in teaching and learning process implying that the conceptual change has been a major research area in science education (Duit and Treagust 2003). Posner and Strike's work described that a change can be taken place when disequilibrium must arise in the form of dissatisfaction with the current concept, intelligibility, plausibility, and fruitfulness of the new concept (Strike and Posner 1985). The key criterion for distinguishing between radical and weak restructuring for conceptual change is whether the concepts central to the theories are intertranslatable (Carey 1986). Thagard (1992) built a model which described various types and different degrees of scientific conceptual changes involving kind-relations (birds, mammals, and reptiles are all kinds of animals), part-relations (birds have feathers and beaks), as well as relations between concepts, and rules that link concepts (whales eat sardines), which are in turn parts of the concept itself. Moreover, Dykstra et al. (1992) organized types of conceptual change into a taxonomy, and this taxonomy is characterized by unique changes in the representation from preconception to postconception and by unique features of the strategies which seem to induce the type of conceptual change represented by those categories: differentiation, class extension, and reconceptualization. As researchers began to explore into how to help students move from their alternative notions to the more scientifically accepted conceptions, they relied on Piaget's theory of disequilibrium, in which assimilation and accommodation play major roles (Posner et al. 1982; Dykstra et al. 1992). Many instructional models for science learning were grounded on the theoretical aspects of conceptual change for several decades, for examples, conceptual change model (Posner et al. 1982), generative learning model (Cosgrove and Osborne 1985), cooperative learning model (Tao and Gunstone 1999), DSLM (She 2002, 2003, 2004), and model-based inquiry (Stewart et al. 2005).

The DSLM is one of instructional models which promote student conceptual development when alternative concept exists (She 2003, 2004a, b). To implement the DSLM into classroom teaching and learning practice, there are six major stages 
in DSLM (She and Liao 2010): (1) examining the attributes of the science concept to provide information in which essential mental sets are needed to construct a scientific view of the concepts; (2) probing students' misconception on the concept; (3) analyzing for mental sets in which the students lack to pinpoint which and how many particular mental sets students lack for restructuring the science concepts based upon the first pair of DSLM theory; (4) designing dual-situated learning events including the ideas of second and third duals of DSLM; (5) instructing with dual-situated learning events to provide students an opportunity to make predictions and provide explanations before and after the event, and to further explain why they changed their conceptions or retained their original conceptions and (6) instructing with challenging situated learning event to provide an opportunity for the students to apply the mental sets they have acquired to a new situation, ensuring that successful conceptual change to occur. According to She's idea of DSLM, any type of instructional activity, such as analogy, modeling, discrepant events, and inquiry activities, could be applied as long as they provide students with opportunities to visualize what actually happens in order to reconstruct new mental sets. However, the instructional activity used in DSLM should be emphasized to enable students make predictions, acquire information, generate explanations, and construct a more scientific view of science concepts (She 2004a, b). This is an alternate plausibility to challenge teachers' pedagogy in order to facilitate student's radical process of conceptual change, and there is a chance to design and consider which pedagogical approach would result in the more successful promotion of conceptual change in science among students.

\section{Application of computer simulation for conceptual change}

Computer simulations have become increasingly powerful and available to science teachers over the last three decades. Instructional computer simulation is recognized as a cognitive visualized tool for effective support of student learning in science by presenting dynamic theoretical or simplified models of real-world components, phenomena, or processes, and enlarging students to observe, explore, recreate, and receive immediate feedback about real objects, phenomena, and processes. As such, simulation-based conceptual learning tools were utilized to support activities of observation, and reflection helps in facilitating the learning of abstract concepts (Chen et al. 2011; Colella 2000; de Jong and Van Joolingen 1998) and providing real-time data displays related to a dynamic phenomenon and information on how certain parameters change synchronously to facilitate higher-level thinking (de Jong and van Joolingen 1998; Ronen and Eliahu 2000). Based on visual-aids learning with computer simulation, its visualized features facilitate the integrated cognitive process of new knowledge and existing knowledge, and improve conceptual understanding in scientific phenomena (Cook 2006; Wu and Shah 2004).

In the field of conceptual change research, simulation-based learning environments are appropriate for manifesting conditions of conceptual change (Chen et al. 2013). Previous research has demonstrated the effectiveness of computer simulations in students' conceptual change. Researchers found that computer simulation works with remedial by producing change to the alternative conceptions held by 
learners (Bell and Trundle 2008; Jimoyiannis and Komis 2001; Muller et al. 2008; Windschitl and Andre 1998; Zacharia and Anderson 2003); improves the performance of gaining intuitive domain knowledge, i.e., more qualitative knowledge than formalized knowledge (de Jong et al. 1999; Veemans et al. 2006); and results in obtaining a more theoretical focus and developing a more coherent understanding of the concepts (Winberg and Berg 2007).

Clearly, the effectiveness of computer simulations is closely connected to the pedagogy through which they are employed (Flick and Bell 2000). Computer simulation has significant potential as a supplementary tool for effective conceptualchange learning based on the integration of technology and appropriate instructional strategies. There are several educational values that computer simulation adds into science learning activities (Hennessy et al. 2006), especially in activity type of inquiry-based science. Inquiry-based learning with computer simulations is generally seen as a promising area for science learning and instruction to foster the obtainment of knowledge and the communication among science ideas in science teaching and learning. In addition, simulation-based inquiry allows students to change variable values and observe effects to form scientific conclusions. Through this process, students discover principles, rules, and characteristics of scientific phenomena (Veemans et al. 2006), implying that they could change their conception when alternative scientific concept exists.

The role of simulation-based open inquiry in DSLM

Inquiry is an educational or learning process which is concerned about the cognitive development of the learner and constructivist ideas of nature of science (Hofstein and Lunetta 2004; Hofstein and Mamlok-Naaman 2007). Inquiry learning has its origins in the practices of scientific inquiry and places a heavy emphasis on engaging students in the investigation of scientifically oriented questions, perform active experimentation, formulate explanations from evidence, evaluate their explanations in light of alternative explanations, and communicate and justify their proposed explanations (American Association for the Advancement of Science [AAAS] 1993; National Research Council 2000) as a set of interrelated processes for developing a rich understanding of concepts, models, theories, and principles (Kuhn et al. 2000). However, more and more evidences indicate that structured inquiry - the highly structured laboratory practices that provide questions, theory, experimental, and analytic procedures-is not sufficient in developing scientific thinking (Zion and Sadeh 2007). This type of inquiry investigation produces a robotic style of thinking that is less effective than teaching deductive reasoning, detailed in-depth thought processes, and logic (Srisawasdi 2012). According to the evidence, engaging learners into a more flexible way of scientific inquiry through conducting laboratory experiment has been given more emphasis in recent science education. Recently, the meaning of open inquiry is not quite clear yet, and inquiry practitioners are still discussing about its characterizations. On the other hand, Buck et al. (2008) described open inquiry in a way that can be used by both secondary school practitioners and university researchers contexts. They described the learning process of open inquiry as an investigation where instructor provides the inquiry 
question or problem and basic background, but the remaining characteristics are left open to the student, wherein learners have to develop their own procedure, analysis, communication, and conclusions to address an instructor-provided question (Buck et al. 2008).

In order to feature DSLM as described by She (2004a, b; She and Liao 2010), the active learning process of open inquiry and characteristics of computer simulation could be embodied in order to feature the process of conceptual change by DSLM. In educational practice of DSLM, Lee and She (2010) pointed out three main steps of teaching practice in the design of DSLM: (1) each dual-situated learning event begins with a driving question, which is targeted to the alternative conceptions commonly found in students; followed by the driving questions: (2) various activities such as graphic and textual illustrations, simulated animations, simulated experiments, and analogies are provided. The design of these activities must create dissonance, challenge students' ontological and epistemological beliefs, and help the students reconstruct the mental sets they lack; and (3) the same driving question is asked again, and students are required to provide an answer with explanations for what happens in the events. As such, simulation-based open inquiry could be implemented into the teaching practice by engaging students in these events: (1) stimulating curiosity and interest by open-ended inquiry question, which is supported by simulation; (2) providing essential theory and basic scientific background to foster testable hypotheses generation, which is supported by simulation; (3) providing opportunity to design experiment and then independently perform the experiment with simulation, by changing conditions and parameters in order to see what actually happens; (4) allowing for independent analysis of data to verify the visualized phenomena; (5) organizing a class communication by presenting the results and then discussing the main idea together; and (6) assisting the students on how to draw a conclusion based on evidence in order to reconstruct new mental sets.

In this study, the researchers employ simulation-based open inquiry activity by embedding into DSLM as a teaching method in order to instruct students' inquiry learning process and enhance their development of conceptual understanding in light refraction.

\section{Purpose}

Based on the above mentioned rationale, the goals of this study were to investigate students' conceptual change for light refraction concepts delivered in DSLM incorporating a simulation-based open-inquiry context. According to the goals, students' conceptual understanding of light refraction, patterns of conceptual change involving the light refraction, and students' cognitive mechanism of conceptual change were investigated. Specifically, the following questions were answered:

(1) Do the students engaged in simulation-based open inquiry with DSLM perform significantly better by conceptual understanding about light refraction?

(2) How were the students' concepts changed after participating in simulationbased inquiry with DSLM context? 
(3) How does simulation-based inquiry with DSLM affect students' cognitive mechanisms of conceptual change?

\section{Methods}

Research design

The purpose of the present study is to investigate the effects of simulation-based open inquiry with DSLM context on secondary school students' conceptual understanding, and conceptual change in a physics course. To broaden perspectives and understanding of the study phenomenon mentioned, the mixed research methodology or mixed-method research with a combination of quantitative and qualitative research methodologies in a research study (Cresswell 2003) was selectively employed in order to answer the research questions. The concurrent embedded strategy of the mixed research methodology was conducted by embedding qualitative research methodology within quantitative research methodology. For this study, phenomenological research methodology was embedded into one-group pre-, post-, and retention quasi-experimental design, in which they addressed different research questions, as shown in Fig. 1.

Study participants

A total of 40 student-respondents in their eleventh grade, age ranging from 17 to 18 years in a local public school at the northeastern region of Thailand participated in this study. They were attending a physics course for basic education level. With an informal interview with the regular class instructor before starting the experimental study, the result indicated that all of them have satisfactory skills on basic computer and information and communication technology, but they have no experience yet using a computer simulation in physics learning. In an addition, they have never experienced having open-ended learning process of scientific

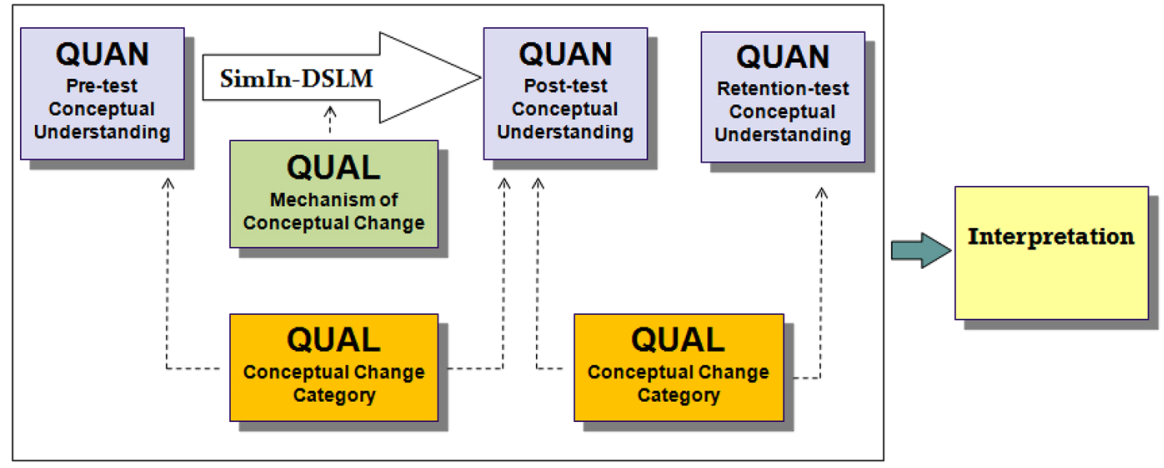

Fig. 1 Structure of research design of the study 
inquiry in any formal class. This implied that they are heterogeneous before interacting with the experimental study.

Learning materials

\section{Design of conceptual learning events on light refraction using simulation}

The design of the conceptual learning events on light refraction was based on the six stages of the DSLM as described earlier. In the first stage, conceptual attributes of light refraction were carefully examined to indicate which essential mental sets were needed for constructing a more scientific view of these concepts. According to this step, a concept map associated to light refraction phenomenon was constructed in hierarchical order of attributes. After the attributes and essential underlying concepts were identified by the researchers and three experts, three mental sets were set up involving refraction at a boundary, Snell's law, and total internal reflection. In the next stage, three conceptual open-ended question items were constructed on the basis of the conceptual attributes in consultation with the three experts. To elicit students' understanding of these concepts, 150 of the eleventh graders were asked to explain three situations about the line of sight: (1) a straw appears normally (no bend) when it is put into a container; (2) a straw appears to bend at the surface of liquid which is held in a container; and (3) a straw disappears at the surface of liquid which is held in a container. The students' written responses were analyzed, and the analysis of students' beliefs about refraction at a boundary showed that $56.77 \%$ of students perceived that, when light travels from air to glass, no distortion happens. About $62.58 \%$ of them believed that there is a reflection of light when an image of pencil appears to be broken. Moreover, $67.74 \%$ of them believed that it is impossible that, when a half of the pencil is placed in a container, it would disappear. Theses students' alternative conceptions need to be changed into scientific conceptions. In the third stage, the researchers identified mental sets of light refraction which students lack in order to design a series of dual-situated learning events for conceptual change including their alternative and incomplete scientific conceptions. According to the results obtained in the second stage, about 78.71, 90.32, and $91.23 \%$ of students lacked the mental sets of refraction at a boundary, Snell's law, and total internal reflection. In the fourth stage, the mental sets which students lacked were categorized by these three conceptual topics, i.e., refraction at a boundary (C1), Snell's law (C2), and total internal reflection (C3), and then the following series of four dual-situated learning events were developed, as shown in Fig. 2:

(1) Boundary behavior of light refraction: identify terminology of refraction of light, where refraction is the bending of a wave when light passes across the boundary between two mediums, and it is caused by a change in the speed of the light wave upon crossing the boundary.

(2) Angle of refraction and Snell's law: identify the change of angle of refraction, where the angle of refraction is the measurable quantity that indicates the amount of refraction taking place at any boundary. 

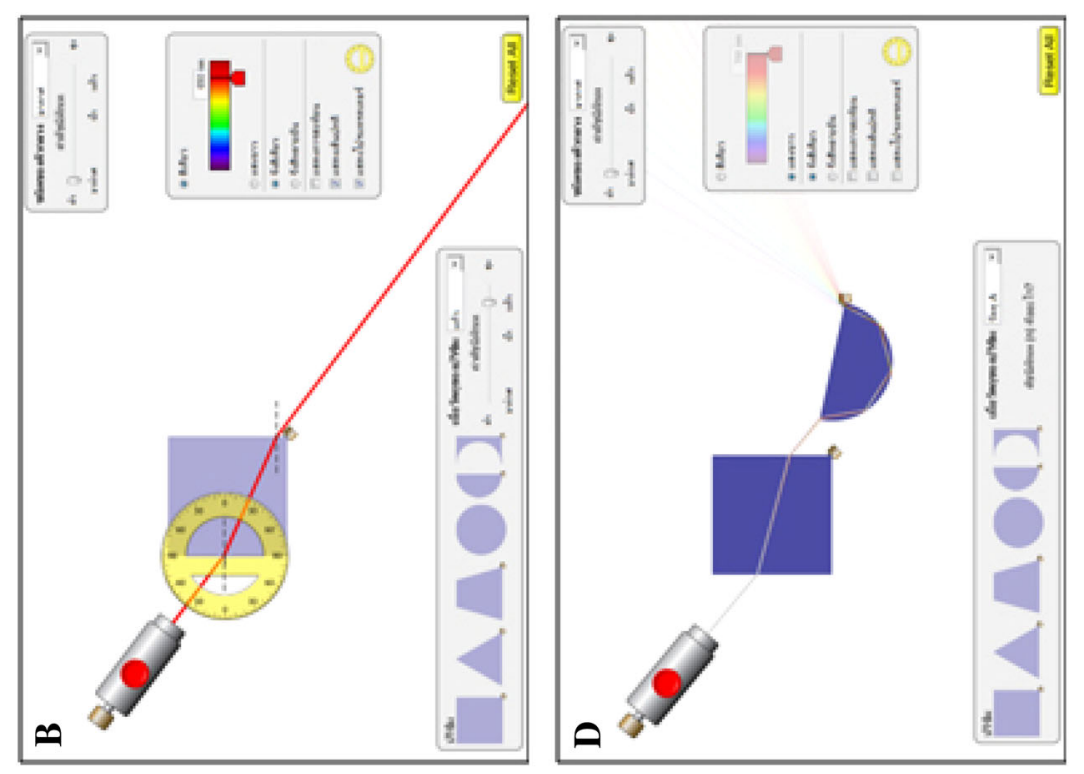

च.
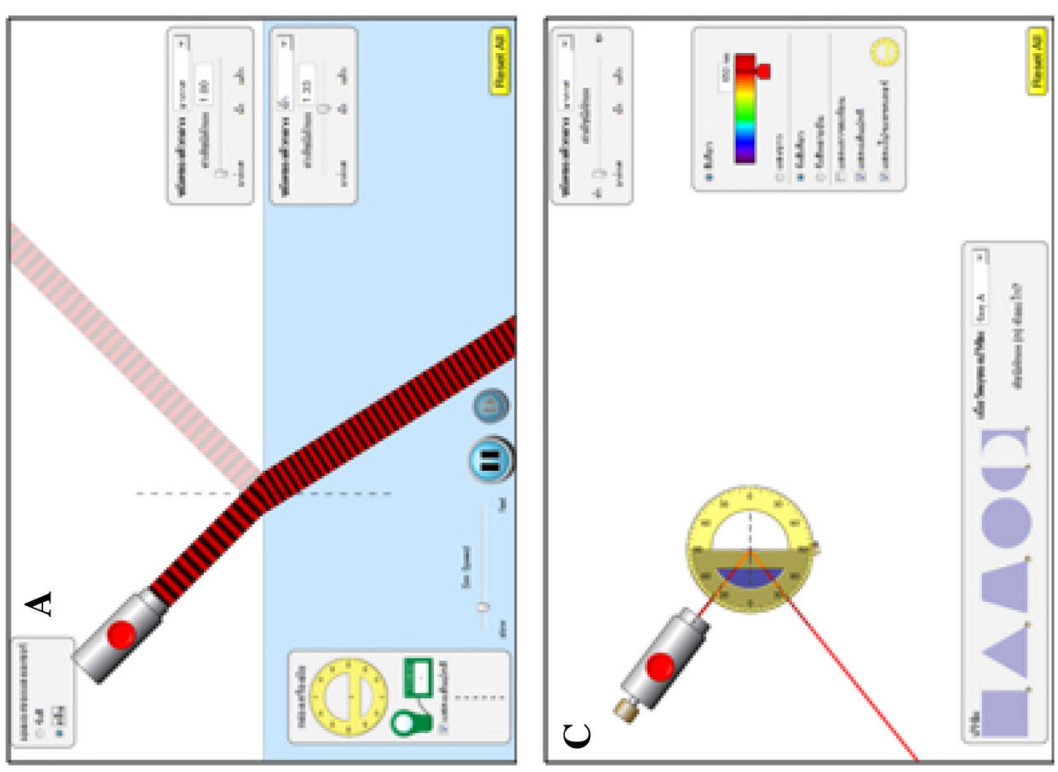

苛

.

苂.

至劳

ㅇํㄹ

焉

ตี

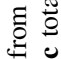

$\ddot{3}$

灵

응

若

䒝芯

\&.

론

这

氧过

莺

这

ง

㝵造 
(3) Total internal reflection of light: identify the connection between light reflection and light refraction, when light wave passes across the boundary of two mediums without refraction because the total amount of incident light was reflected off at the boundary.

(4) Indexes of refraction and dispersion of light by prism: identify an indicator of the optical density of a material, where the index value is a number that indicates the number of times that a light wave would be slower in that material than when it is in a vacuum.

The four dual-situated learning events specifically focus on students' alternative conceptions and incomplete scientific conceptions of light refraction. In order to facilitate students' learning of light refraction concept through the designed learning events as mentioned previously, an interactive computer simulation on refraction of light, named Bending Light, obtained from Physics Education Technology (PhET) research group, was used as a cognitive tool for students. It is clear that students' common alternative conceptions of refraction of light are due to the invisibility of the amount involved and their nature, making it more difficult to construct concepts related to refraction of light. Moreover, many physical situations of light wave refraction are unusually observed, and it makes discrepant events bothering human common senses. Therefore, the design of conceptual learning events on light refraction through the use of computer simulation supports students with visualizations of the refraction of light phenomenon to help them build more scientific views of light refraction concepts. For example, one part of the refraction of light simulation allows students to interact with the simulation to understand the introduction of light refraction in which student would be learning how light refracts, when through the same medium and when through a different medium as shown in Fig. 2. In the fifth stage, simulation-based open inquiry was used to follow the first three dual-situated learning events (see Fig. 2). This pedagogy begins with an open-ended driving question targeted to alternative conceptions commonly found in students. To assist the process of hypothesis generation addressed the question, essential scientific backgrounds are provided to students. Then, students are required to perform generating testable hypotheses, designing an investigative experiment with simulation, analyzing the data, communicating results of experiment, and drawing a conclusion based on evidence and the testing of the hypotheses (see Table 1). In the final stage, the fourth dual-situated learning event was implemented to challenge students with the mental sets they have acquired to a new situation (see Fig. 2). This challenging-situated learning event served to explore whether students had acquired the mental sets required for understanding the concepts of light refraction.

Learning activities of light refraction by simulation-based open inquiry with dualsituated learning model

An example of learning activities of light refraction by simulation-based open inquiry with DSLM is provided to show how dual-situated learning events can help students to construct and reconstruct their conceptual understanding of refraction of 
Table 1 Components of simulation-based open inquiry with dual-situated learning model teaching method for students' conceptual learning in boundary behavior of light refraction and its learning process

Components of simulation- $\quad$ Examples of learning process
based open inquiry

Pre-lab

Open-ended inquiry question

Scientific background/ information

Lab practice

Procedure/design

Data and result analysis

Post-Lab

Result communication

Conclusion
Teacher provides an open-ended inquiry question: "What will happen if light waves strike a boundary of mediums?"

Teacher induces collaborative discussion toward the definitions and pictorial diagram of incident ray; reflected ray; refracted ray; normal line; angle of incident; angle of reflection; angle of refraction

Students design their own scientific experiments and then interact with Bending Light simulation for collecting the experimental data, for examples, by changing medium index of refraction and angle of incident in order to observe reflected and refracted ray direction, angle of reflection and refraction and so on, and to measure the angles as well

After the interacting with simulation, students make a decision to analyze obtained experimental data from their own design and interpret it into results, for examples, calculation of arithmetic mean and standard deviation in order to use inferential statistics for comparing the difference or use graphical charts for presenting the result

Students have to select the way to present, communicate, and discuss the meaning of data and experimental results to others, for an example, writing experimental question, experimental design, results, and discussion on a newsprint paper and then present to the class. In addition, they might use the Bending Light simulation to demonstrate and explain their results

Students have to collaboratively make a relationship between each group results and then draw it into a conclusion as the best answer to the provided inquiry question. For an example, teacher induces each group make a conclusive answer by using an integration of their own and other results

light concepts. In an addition, to enhance students' scientific thinking and authentic practice of science, laboratory activity of open-inquiry science was employed into the learning activities. This simulation-based open-ended inquiry was aimed to afford students' active conceptual learning in the concept of boundary behavior of light refraction and its bending condition when light waves pass across the boundary between mediums. Moreover, the activity was purposely designed to help students construct their scientific thinking and understanding about how different reflection and refraction of light from each other are and what happens when light wave passes across the boundariesy of same or different mediums. The learning process of simulation-based open inquiry with DSLM started with an open-ended inquiry question about the behaviors of light reflection and refraction provided by teacher. Scientific background associated with the phenomenon, not the answer of the 
inquiry question, was discussed and presented to the students. Afterward, the students were exposed to the laboratory materials, as seen in Fig. 2a. Each student group was assigned to design their own experiment using the provided materials in order to test their set-up hypothesis and then answer the inquiry question. They were, also, needed to make decision for analyzing the obtained experimental data and interpreting the result. In addition, each group was assigned to communicate findings from their experiments to the class, and then collaboratively make conclusions about the experiment with other students and teacher. As described, all components of open-inquiry science laboratory are presented in Table 1.

\section{Experimental procedures}

All of the students received context of simulation-based open inquiry with DSLM for four two-hour class periods. All students took $45 \mathrm{~min}$ to complete a series of seven open-ended conceptual question items as pretest. Then, the students received 15-minute orientation of the purposes of the present study and the learning steps of the simulation-based open inquiry by the researchers. Following this, the researchers gave an orientation about open-inquiry learning process by demonstrating (a) how to find out and determined variables from an open-ended inquiry question; (b) how to generate an experimental hypothesis regarding the variables; (c) how to design an experiment that accounted the generated hypothesis, and then record experimental data and analyze the obtained data in order to examine the acceptance or rejection of the hypothesis; and (d) how to make a scientific conclusion based on real experimental datasets, for $40 \mathrm{~min}$ and 20-minute introduction sessions on the Bending Light simulation by presenting (a) important experimental parameters; (b) interactive components; (c) features; and (d) how to work with the simulation. During the introduction, students were asked to practice following the teacher's demonstration during the simulation instruction process in order to observe what is going on for the simulation and discuss with the students. Next, students performed the 4-hour learning activity. They were encouraged to explore the given open-ended inquiry question on light refraction by conducting open-inquiry learning process, as shown in Table 1, using the simulation-based learning material, as illustrated in Fig. 2. During the learning activity, one group of students was selected as a representative case by the teacher, and a series of video and audio recording were fully taped by two teacher assistants who were available in the classroom for supporting the students' learning activity. The audio-taped transcripts were used for the bulk of the transcripts, and the videotapes provided additional information detailing the students' expressing their conceptual knowledge as they reacted to the learning program. Figure 3 displays an example of students' interaction with the context of simulation-based open inquiry. Finally, a 45-minute posttest was applied after the experiments were completed. For the posttest, the same open-ended conceptual question items were administered to the students again for exploring current status of their conceptual understanding. Moreover, eight weeks after the instruction, a 45-minute retention test was administered to them in a regular classroom. 

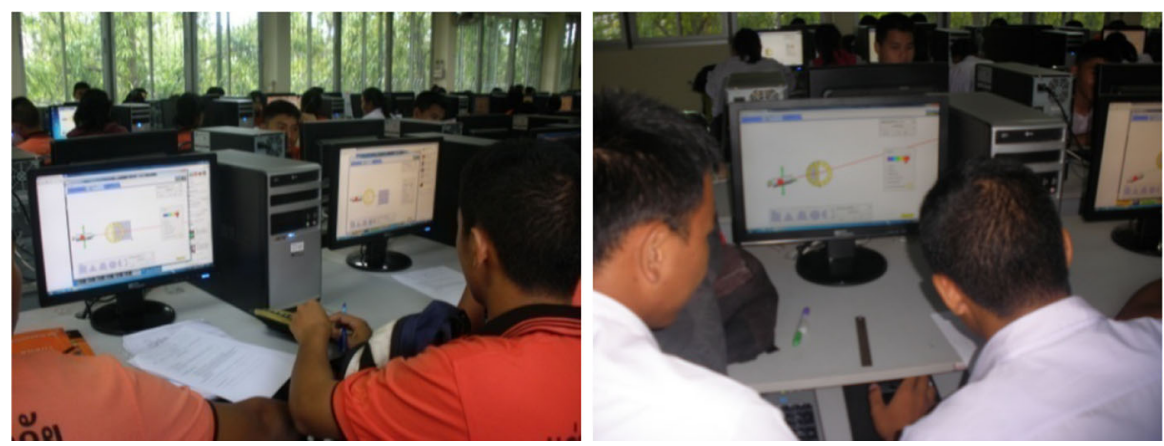

Fig. 3 An illustrative example of classroom learning activity through simulation-based inquiry with dual-situated learning model

\section{Analysis of data}

In the study of contemporary social science of education, content analysis and discourse analysis are systematic research techniques of textual analysis, which are used for better understanding of the reality of educational phenomena. Content analysis has been described as a technique of textual analysis, which is characterized by a concern with being objective, systematic, and quantitative description of the manifest content of communication (Berelson 1952; Kassarjian 2001). The analytic technique of content has been used for a variety of textual analyses by which latent contents of spoken or written text are determined (Krippendorff 1980; Weber, 1985). Based on content analysis, this technique is the study of the text itself, but not of its relation to its context, the intentions of the producer of the text, or the reaction of the intended audience (Hardy et al. 2004) by comparing, contrasting, and categorizing a set of data in order to provide a quantitative treatment of issues of quality (Koehler et al. 2007). A number of researches have been conducted by means of content analysis (e.g., Dias and Diniz 2014; Gerber et al. 2008; Jimoyiannis and Angelaina 2012; Koehler et al. 2007; Schifter et al. 2012). Riffe et al. (1998) stated that content analysis can be reduced to three essential steps: (1) identifying representative samples of the communication; (2) creating a protocol for identifying and categorizing the target variables such as rubrics and protocol for coding; and (3) scoring to describe the target variables or coding to identify relationships between variables. In addition, the content analysis can involve both numeric (quantitative) and interpretive data analyses (qualitative), or combinations of both (Koehler et al. 2007).

In this study, the primary methodology, used for the analysis of students' written responses to the open-ended question items, represented their conceptual understanding, [both pre-, post-, and retention-test data was quantitative content analysis.] In keeping with the three-stage process outlined above, the researchers began with repeatedly read the students' written responses [both pre-, post-, and retention-test]. The next step was the development of a general conceptual understanding rubric that applied to all items and then item-specific rubrics consistent with key concepts 
of light refraction and then actually scoring the data in order to evaluate conceptual quality of students' understanding. Based on the rubrics, the scores in the conceptual understanding rubric ranged from 0 to 3 , with 3 being the highest, for the first item, and its rubric scores were the following: 0 demonstrates no attempt and no conceptual understanding; 1 demonstrates limited conceptual understanding and/or having any significant error; 2 demonstrates some conceptual understanding, but was incomplete; and 4 demonstrates conceptual understanding. For another six items, the scores in the conceptual understanding rubric ranged from 0 to 2 , with 2 being the highest and their rubric scores were the following: 0 demonstrates no attempt, and no conceptual understanding; 1 demonstrates limited conceptual understanding, and/or having any significant error to some conceptual understanding, but was incomplete; and 2 demonstrates conceptual understanding. These rubrics were constructed and done based on consensus by the two researchers, and the two teacher assistants, ensuring that the rubrics were properly evaluated and relevant to the specific concepts of light refraction. The qualitative data collected from students' responses to the items were quantified into a numerical score based on the item-specific rubrics. This numerical score represented the students' level of conceptual understanding about light refraction. In order to use statistical analysis for students' conceptual understanding scores, the normal distributions of data were not met for their scores. Thus, nonparametric statistics of Friedman test and Wilcoxon sign-ranked test were used to examine significantly differences for their conceptual understanding scores on the pre-, post-, and retention tests.

In addition to the quantitative content analysis, the qualitative changes of students' conceptual understanding between pre-, post-, and retention tests were interpreted and then quantified into five categories based on She and Liao's (2010) ideas including (a) Progress (PG) — to what extent the student's conceptions improved; (b) Maintain-correct (MTC) — to what extent the student's conceptions were maintained correctly; (c) Maintain-partial correct (MTPC)—-to what extent the student's conceptions were maintained as partially correct; (d) Maintain-incorrect (MTIC) - to what extent the student's conceptions were maintained as partially incorrect; and (e) Retrogression (RTG) — to what extent the student's conceptions retrogressed. This section of analysis provides a sequential representation of the change of students' conceptual understanding as elicited in students' written responses. Moreover, this method of analyzing cognitive structure based on students' written responses has suggested that this analysis is a useful and valid method of representing students' conceptual frameworks in science and quantity of conceptual change (She and Liao 2010). The quantity of conceptual change was described by respective percentages for PG, MTC, MTPC, MTIC, and RTG from pretest to posttest, and from posttest to retention test.

In contrast to content analysis, discourse analysis is a research technique for analyzing social phenomena, which is qualitative, interpretive, and constructionist in order to understand the meaning of social reality and uncover the way in which reality is produced (Phillips and Hardy 2002). Discourse analysis becomes an effective technique for studying how talk and texts are used to perform social actions and the varying resources that people draw on in the course of those actions (Hanrahan 2005; Potter 2003). This research technique, in the context of classroom, 
tends to focus more on the use of spoken language during communication than that of written responses because it reveals specific ways of acting and interrelating; specific ways of representing; and specific ways of being (Fairclough 2003). The technique of discourse analysis is the study on the vital link between text and the broader social context of its production that consists three interrelated processes of analysis: (1) description of the text; (2) interpretation of the relationship between the text and the social processes of the interaction; and (3) explanation of the relationship between the social processes and the social context (Fairclough 2001). A number of researches have employed discourse analysis to investigate classroom phenomena (e.g., Hannon and Bretag 2010; Russ et al. 2008; Sing and Khine 2006)

In an effort to qualitatively explain cognitive process of conceptual change during students' interaction of simulation-based open inquiry with DSLM, for this study, the classroom verbal protocol, the transcripts of video and audio, was analyzed by discourse analysis. The researchers started with transcribing the video and audio recordings and repeatedly read the transcripts while identifying and labeling the linguistic features in the protocol. By this step, some manageable chunks of verbal protocol for illustrating the cognitive process were found for the interpretation of the classroom setting. Then, the role of the analyst is to interpret the cognitive process of conceptual change, as they manifest in protocol, based on a taxonomy of conceptual changes (Dykstra et al. 1992): (a) Differentiation-wherein new concepts emerge from the existing, more general concepts; (b) Class extension-wherein existing concepts considered different are found to be cases of one subsuming concept; and (c) Reconceptualization-wherein a significant change in the nature of and relationship between concepts occurs. The categories in this taxonomy are characterized by unique changes in conceptual representation from pre-conception to post-conception and by unique features of the strategies which seem to induce the type of conceptual change represented by those categories (Dykstra et al. 1992). Finally, the researchers explained the study phenomena by redescribing cognitive process of conceptual change made by students that it was an impact of the simulation-based open inquiry with DSLM. Through these steps, the students' cognitive processes of conceptual change were captured.

In conclusion, this study was designed to better understand on the development of scientific understanding in particular context setting of simulation-based open inquiry with DSLM. An integration of content analysis and discourse analysis was, therefore, used to investigate impact of the context on students' conceptual understanding of light refraction. This integration forms an essential extension of the analysis method of textual data within social science research (Wilson, 2003), and these two methods can be seen as complementary and even mutually supportive in the exploration of social reality (Hardy et al. 2004; Neuendorf 2004). The quantitative content analyses provides fruitfulness going beyond the surface content of the transcripts toward the identification and analysis of latent variables (i.e., students' conceptual understanding and conceptual change). Moreover, discourse analyses were conducted where evidence of convergent conceptual change process emerged. These evidences provide additional empirical validation in favor of the development of conceptual understanding about light refraction. As such, both analytic methods used in this study complement each other in order to provide more 
comprehensive picture in the nature of "conceptual change" in simulation-based open inquiry with DSLM.

\section{Results}

Analysis of conceptual understanding score

In order to explore the effects of the simulation-based open inquiry with DSLM on the students' conceptual development of light refraction, results of statistical analysis using nonparametric Friedman test are shown in Table 2.The results from Table 2 indicated that there was a statistically significant difference among overall pre-, post-, and retention-test scores of conceptual understanding on light refraction, $\chi^{2}(2)=44.00, p<0.01$. The result indicated also that the students' conceptual understanding showed significant improvement after participating with the simulation-based open inquiry with DSLM (from 5.05 to 11.80), but they made a slight decrease from posttest to retention test (from 11.80 to 10.50). Post hoc analysis with Wilcoxon signed-rank test was conducted with a Bonferroni correction applied, and the results indicated that the students made a great progression of their conceptual understanding of refraction of light considering from pretest to posttest and the posttest score was significantly greater than the pretest $\left(Z=-5.22, p_{\text {(post }>\text { pre) }}<0.000\right)$. Moreover, a great progression of their conceptual understanding was found on a difference between pretest and retention-test scores, and the retention-test score was significantly greater than the pretest $\left(\mathrm{Z}=-5.33, p_{\text {(post }>\text { pre) }}<0.000\right)$. However they also made a slight decrease of conceptual understanding scores from posttest to retention test and its difference was also significantly $\left(\mathrm{Z}=-2.19, p_{\text {(post }>\text { pre })}=\right.$ $0.028)$.

\section{Analysis of conceptual change category}

The effect of simulation-based open inquiry with DSLM on students' conceptual change of light refraction was also investigated in this study. Five categories (PG, MTC, MTPC, MTIC, and RTG) were used to interpret a transitional change of students' conceptual understanding from pretest to posttest and posttest to retention test, respectively. The percentages of the quantity of conceptual change on the transitions were presented in Figs. 4 and 5.

Table 2 Statistical results of Friedman test and Wilcoxon sign-ranked test on conceptual understanding scores

\begin{tabular}{llllll}
\hline $\begin{array}{l}\text { Test of conceptual } \\
\text { understanding }\end{array}$ & $\begin{array}{l}\text { Mean } \\
(\text { Max. }=15)\end{array}$ & Mean rank & $S D$ & Chi square & $\begin{array}{l}\text { Post hoc } \\
\text { comparison }\end{array}$ \\
\hline (a) Pretest & 5.05 & 1.18 & 3.02 & $44.00^{*}$ & (b) $>(\text { a })^{*}$ \\
(b) Posttest & 11.80 & 2.55 & 2.84 & & (b) $>$ (c)* \\
(c) Retention test & 10.50 & 2.28 & 2.95 & (c) $>(\text { a })^{*}$ \\
\hline
\end{tabular}

$* p \leq 0.01$ 


\section{Pre-test - Post-test}

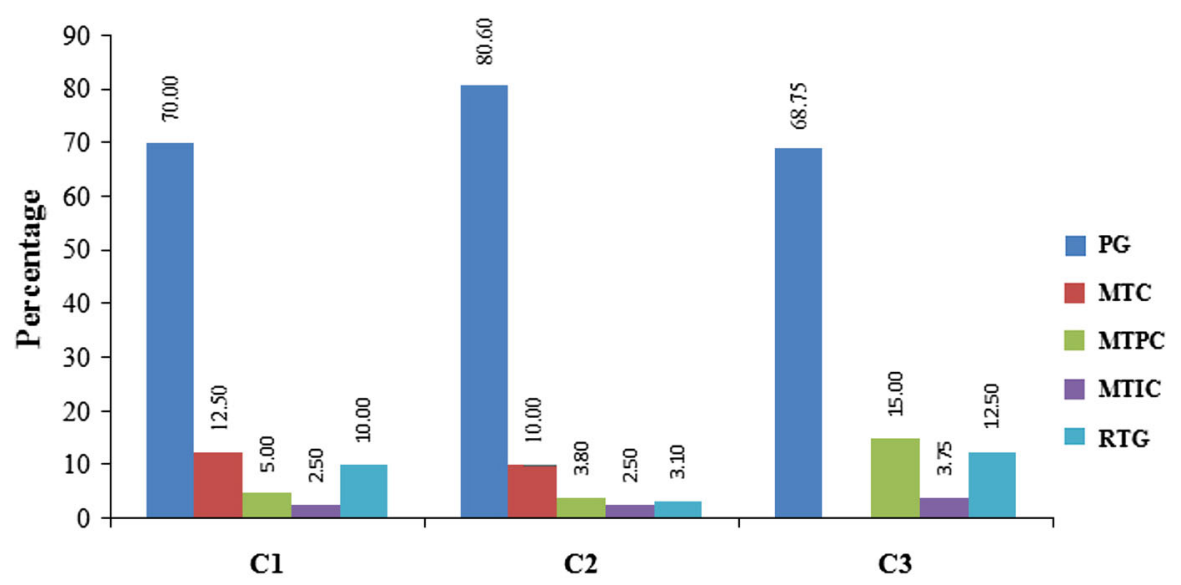

Concepts of Light Refraction

Fig. 4 Distribution of conceptual change across three concepts (i.e., refraction at a boundary $(C 1)$, Snell's law (C2), and total internal reflection $(C 3))$ from pretest to posttest

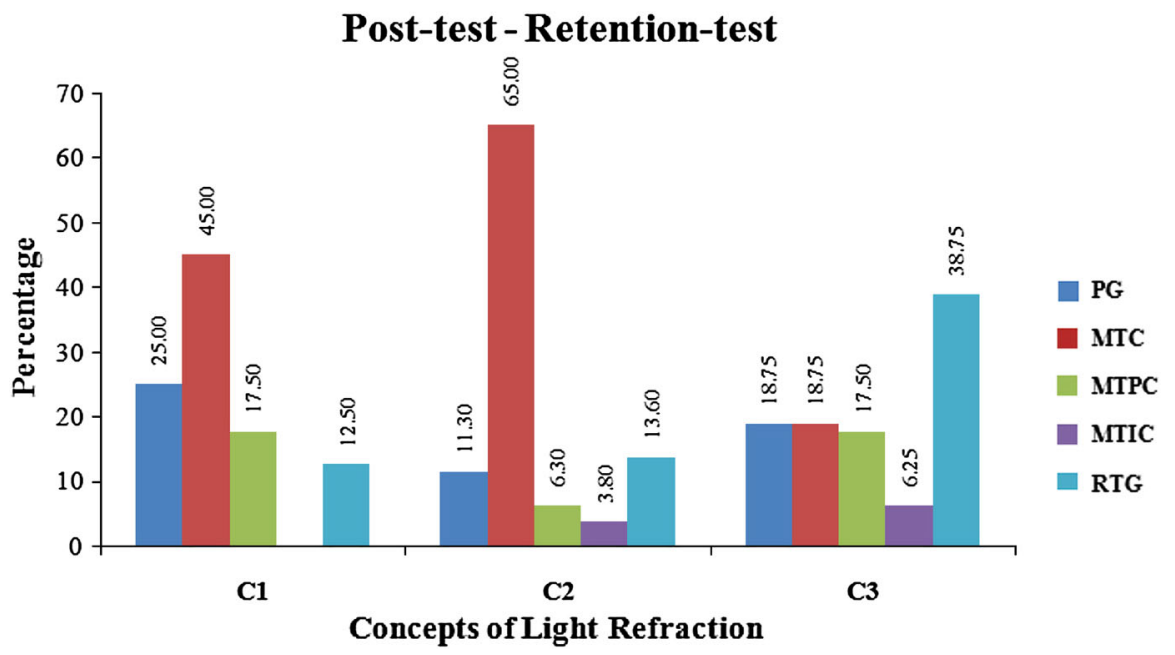

Fig. 5 Distribution of conceptual changes across three concepts (i.e., refraction at a boundary $(C 1)$, Snell's law $(C 2)$, and total internal reflection $(C 3))$ from posttest to retention test

As seen in Fig. 4 for pretest to posttest, the percentage of the Progress (PG) category was higher than any other category across most of the three concepts, and C2 concept was the highest. The percentages for Progress (PG) ranged from 68.75 to 80.60 , Maintain-correct category (MTC) ranged from 10.0 to 12.50 except no found on C3 concept. The Maintain-partial correct (MTPC) and Maintain-incorrect 
(MTIC) were found across three concepts. The percentages for MTPC and MTIC ranged from 3.80-15.00 and 2.50-3.75 respectively. The highest percentage of Retrogression (RTG) was found on C3 concept and the percentages of RTG ranged from 3.10-12.50.

Figure 5 displays the percentages of the quantity of conceptual change from posttest to retention test across the three concepts of light refraction phenomena.

As seen in Fig. 5, there is the percentage of change for students' understanding of refraction from posttest to retention test. The percentage of Maintain-correct category (MTC) was higher than those of other categories for $\mathrm{C} 1$ and $\mathrm{C} 2$ concepts, and the highest was 65.00 in $\mathrm{C} 2$ concept. The Progress (PG) category was found across the three concepts, with the percentages for PG ranging from 11.30 to 25.00. Similarly, Maintain-partial correct (MTPC) were also found across three concepts and the percentages for PG ranging from 6.30 to 17.50 . The Retrogress (RTG) category was the highest for $\mathrm{C} 3$ concept and found across the three concepts. Maintain-incorrect (MTIC) was the highest in $\mathrm{C} 3$ concept except in the $\mathrm{C} 1$ were not found.

Analysis of cognitive mechanism of conceptual change

\section{Example of discourse protocol \#1}

Using Dykstra et al. (1992) taxonomy of conceptual change process, the following discourse is an example of students' cognitive mechanism of conceptual change during interacting with the simulation-based open inquiry with DSLM. The following protocol excerpt represents the type of Differentiation. This protocol excerpt represented that a more general concept of light refraction emerged from existing informal concepts based on student prior knowledge or pre-experience.

[1] Student A The wave of light always travels in a straight line

[2] Student B Why?

[3] Student A It likes ... The sun light travels straight line in the air into the world. If the light travels in the same medium, the straight line of travelling will always happen

[4] Student B What should be a hypothesis on the inquiry question? (Note: the inquiry question is what would be happen if light waves travel across two different media?)

[5] Student A Umm... It will be changed the travel direction. The direction is not the same line as they move before. I mean... Yes, that is it. The light waves are refracted when they travel through different mediums

[6] Student B Why?

[7] Student A The media have different density

[8] Student B So, The light travel direction is not going to change when they move within a medium, right?

[9] Student A Yes. Let's start the simulation experiment. Following the teacher manual, both materials are going to be "air" and let see what would happen? 
[10] Student B Nothing happened. Let's change the second material to be "water"

[11] Student A Oh wow! It changed

[12] Student B It bends

[13] Student A Yes, it bends closely into the normal line, and we have to mention the media

[14] Student B We have to mention that when light waves travel through different media, they bend, right?

[15] Student A Yes, they do. OK, we have to describe that if light waves travel through different media, the travel direction is going to be changed

[16] Student B How about traveling through the same medium? Did it change?

[17] Student A Exactly, it would not change on the travelling in a medium. OK. Let's make a conclusion for this. When light waves travel across a boundary of the same medium, they are not going to change their travel direction, but the direction is going to be changed by bending to the normal line if they travel across a boundary of different mediums because of their density. Write it on

An example of this protocol excerpt demonstrated the cognitive process of Differentiation for conceptual change occurring in Student A. Initially, the Student A held a great amount of scientific knowledge about property of light wave and medium as highlighted in lines 1, 3, 5, and 7. These are a part of her existing knowledge and prior experience. During their simulation-based open-inquiry learning process with the Bending Light simulation, Student A has visualized the light refraction phenomena with the affordance of Bending Light simulation and realized how the world works on these phenomena as highlighted in lines 11 and 13 . This evidence implied that this probably helps her discover a reason as to why light waves are refracted when they travel across boundaries of different mediums, and

\section{$\begin{array}{lll}\text { Existing Conception } & \text { New-coming Conception } & \text { Refine Conception }\end{array}$}

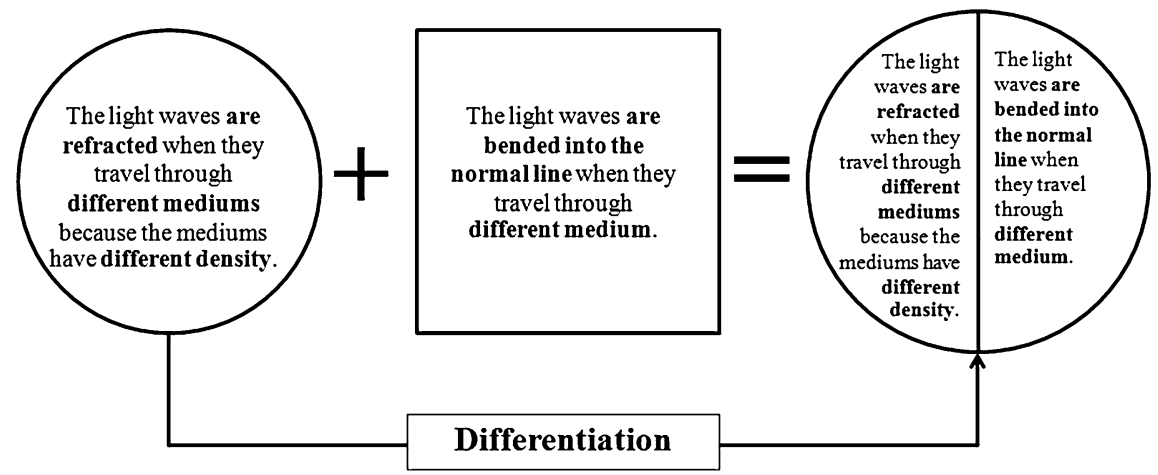

Fig. 6 A diagramatic representation of differentiation cognitive mechanism of conceptual change process 
she has already known that the property of medium plays an important role for the phenomena. Moreover, she found a new knowledge that the light waves are going to bend closely toward the normal line. In the end, Student A could get some more scientific details of the light refraction phenomena into a general view as highlighted in line 17. Figure 6 is used to represent the cognitive mechanism of Differentiation on Student A's conceptual change process during interaction with simulation-based open inquiry with DSLM learning program.

\section{Example of discourse protocol \#2}

The following protocol excerpt represents the cognitive mechanism of Class Extension in conceptual change process. This protocol excerpt represented that a subsuming concept is found as necessary factor in order to better understand the light refraction phenomena based on existing concepts.

[1] Student A What would happen if light waves travel into a prism which is placed in the air? (Note: he read the inquiry question on a lab data sheet)

[2] Student B Is it reflected? Is it going to reflect or refract?

[3] Student A It must be refracted because the light moves into prism

[4] Student B Why?

[5] Student A Because.... Because... This is it reason! (Note: he pointed out the information displayed in the lab data sheet) It is in optical property of light. Refraction is the bending of the light wave when it passes across the boundary between two different mediums

[6] Student B So, because of passing through different medium, it is refraction of light, right?

[7] Student A Yes. The hypothesis is that when light waves travel into a prism which placed in the air, they are going to change their travel direction by bending

[8] Student B OK. Let's do the experiment

[9] Student A Let's make a light travels into a rectangular glass prism and then measure theta 1 (Note: this is angle of incidence) and theta 2 (Note: this is angle of refraction) at the first boundary

[10] Student B The angle of incidence is 30 degree and another is 20 degree

[11] Student A Then, move to measure theta 3 (Note: this is angle of incidence) and theta 4 (Note: this is angle of refraction) at the second boundary

[12] Student B They are 20 and 30 degree, respectively

[13] Student A This time changes the object to be water prism and then repeat the procedures

[14] Student B Theta 1 is 30 degree and theta 2 is 22 degree

[15] Student A Next is theta 3 and 4

[16] Student B They are 22 and 30 degree, respectively

[17] Student A Write a table and fill the data into the table

[18] Student B How to explain the data? 
[19] Student A Let say. The refraction of light relatively depends on types of material which is its medium. The light waves travel into prisms which made by different material, the waves are going to refract differently as well depended on the density of material

[20] Student B Then?

[21] Student A If the light travels through a high density prism, it is going to be refracted in a small degree of angle and the light is going to bend closely into the normal line, and vice versa. That is, the refraction of light is going to be difference related to materials types of prism

This protocol excerpts illustrated the cognitive process of Class Extension for conceptual change occurring in Student A. Initially, the Student A displayed an idea about light refraction in a prism, as highlighted in line 3, but he did not know why it is at the moment. Afterward, he discovered useful information in the lab data sheet, and then he could recall and recognize the reason as highlighted in line 5. During their conducting an inquiry with the Bending Light simulation, Students A and B immediately obtained experimental data from the simulation experiment so that they can directly visualize and measure the angles using a supportive tool in the simulation. Moreover, the simulation could afford their learning so that they can do the experiment many times with different object materials and set up the experiment in a very short time. The obtained data could be used to make them think and then generate an explanation to the studied phenomena, as highlighted in lines 9-17. In the end, Student A can find a subsuming concept after their simulation experiment, that is, the refraction of light is going to be differently related to materials' types of prism as highlighted in line 21 . This extended concept is an important factor in order to better understand the light refraction phenomena. Figure 7 is used to represent the cognitive mechanism of Class Extension on Student A's conceptual change process during interacting with the simulation-based open inquiry with DSLM learning program.

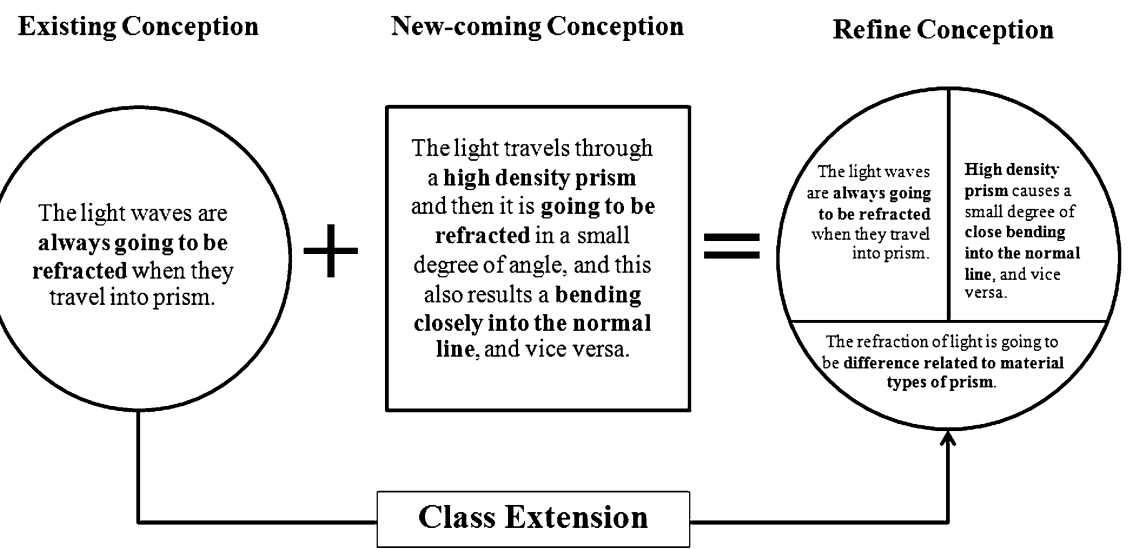

Fig. 7 A diagramatic representation of class extension cognitive mechanism of conceptual change process 


\section{Example of discourse protocol \#3}

In contrast, the following protocol excerpt represents the cognitive mechanism of Reconceptualization in conceptual change process. This protocol excerpts represented that a significant change in the nature of and relationship between concepts of light refraction occurs. For an example, a wrong relationship of knowledge objects in refraction of light phenomenon is replaced with a correct one.

[1] Student A Take a look on this. If both mediums are the air, incidence and refracted ray of light wave are going to...

[2] Student B To be a straight line. (Note: he pointed out shape of the incidence and refracted ray of light wave within an upper medium (air) separated by a normal line)

[3] Student A Yes, the ray is always a straight line and angle of incidence and reflection are always equal. Look at this. This is 50 degree of the angle of incidence and 50 degree of the angle of reflection. (Note: he pointed out the angles within the air medium) Actually, you should take a look at this boundary between two different mediums, i.e., air and water. You should observe this angle (Note: he pointed out the angle of refraction in the water medium) comparing with this angle. (Note: he pointed out the angle of incidence in the air medium)

[4] Student B It is a straight line. Nothing is changed

[5] Student A Really? You should observe by comparing the angles

[6] Student B It is not equal. Oh, it is refracted

[7] Student A Yeah. It changed direction

[8] Student B Oh yes. If the light travels from air into water, the angle of incidence in the air and angle of refraction in the water are not equal value. So, it is different comparing with the upper medium. Anyway, what is this line? (Note: he pointed out the reflection ray of light)

[9] Student A It is a shadow of incidence ray. That is why both angles in the air (Note: both angles were separated by a normal line) are equal at 50 degree, but the angle of refraction in the water is smaller, which is 30 degree

This protocol excerpt illustrated the cognitive process of Reconceptualization for conceptual change occurred in Student B. At the beginning of this protocol excerpts, the Student B displayed an alternative (unscientific) conception that the light waves are not refracted even they travel through boundaries of two different mediums, as highlighted in line 4. Afterward, Student A guided him to observe comparatively between the angle of incidence in the air medium and the angle of refraction in the water medium in the Bending Light simulation, as highlighted in line 5. Student B simultaneously obtained factual data from the simulation experiment, which they could directly visualize and measure the angles by using a supportive tool in the simulation. Finally, Student B was able to realize that this is refraction of light as highlighted in lines 6 and 8. Unfortunately, Student A still held an alternative 
$\begin{array}{lll}\text { Existing Conception } \quad \text { New-coming Conception Conception } & \text { Refine }\end{array}$

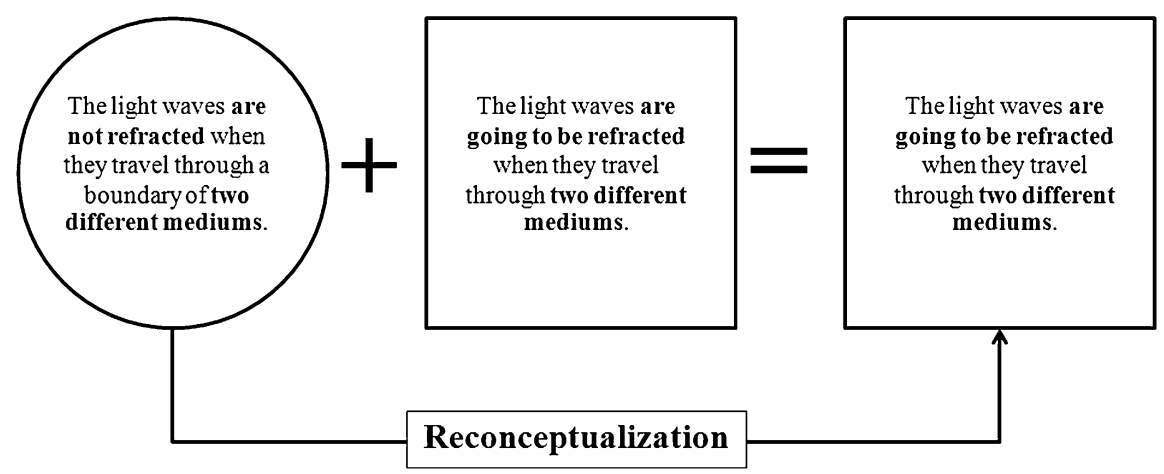

Fig. 8 A diagramatic representation of Reconceptualization cognitive mechanism of conceptual change process

conception about reflection of light that the reflection ray was only a shadow of incidence ray as displayed at the end of this protocol excerpt in line 9. Figure 8 is used to represent the cognitive mechanism of Reconceptualization on Student A's conceptual change process during interaction with the simulation-based open inquiry with DSLM learning program.

\section{Discussion}

This study reports an impact of an innovative teaching method called simulationbased open inquiry with DSLM on promoting students' conceptual understanding of refraction of light by inducing cognitive process of conceptual change. The result shows an increasing of students' conceptual understanding scores from pretest, posttest, and retention test that reached a statistically significant effect across three concepts of light refraction. This finding could be argued that their understanding on light refraction concepts made progress throughout their learning by the teaching method. This indicates that the teaching method successfully helped students reconstruct a more scientific view of light refraction. The result is consistent with the research findings that students performed better achievements with learning from computer simulation (Bell and Trundle 2008; Hsu 2008; Jaakkola et al. 2011; McElhaney and Linn 2011; Olympiou and Zacharia 2012; Renken and Nunez 2013; She 2002, 2003, 2004a, b; She and Liao 2010; Suits and Srisawasdi 2013; Zacharias et al. 2008). A possible explanation for why students made progress on conceptual understanding from pretest to posttest is that the learning program could induce students into the cognitive mechanism of conceptual change. Evidence supports this explanation was illustrated on this study result of the quantity of conceptual change. The result shows that the percentage of the Progress (PG) category was higher than any other category across three concepts of refraction of light from pretest to 
posttest. In an addition, empirical evidences displayed in result of discourse protocol analysis also indicate that the students have changed their conceptions about light refraction by cognitive process of differentiation, class extension, and reconceptualization. This shows the potential of simulation-based open inquiry with DSLM that the students gained more scientific views of their conceptions because of the learning program. These evidences are consistent with those of She $(2004 \mathrm{a}, \mathrm{b})$ that a series of well-designed dual-situated learning events can induce process of conceptual change in classroom instruction. It supports the conclusion that using DSLM is efficient for facilitating students' conceptual changes, and cognitive process of conceptual change can be occur if the instructional approach is supported by well-developed educational models (She and Liao 2010).

Considering the conceptual change result for a transition from posttest to retention test, the quantity of conceptual change shows that the percentage of Maintain-correct (MTC) category are higher than any other category across most of the concepts from posttest to retention test. Furthermore, more than $70 \%$ of their conceptual understanding in $\mathrm{C} 1$ and $\mathrm{C} 2$ concept, by regarding PG and MTC, has been reserved in the status of acceptable scientific conception even two months after the learning program. The result shows a radical change of their conceptual understanding after their interacting with the learning program. This finding could be argued that their conceptual understandings about light refraction revised by cognitive process of conceptual change were maintained within their cognitive structure of conceptual knowledge after finishing the learning program. The argument is consistent with the finding that the DSLM is a well-designed instructional model for facilitating students' conceptual change, and it had great potential to foster a radical conceptual change process in science learning (She 2004a, b). Moreover, this is in correspondence with a claim that conceptual change is not necessarily a slow and gradual process and that conceptual change can be achieved within a short time through the use of DSLM (She and Liao 2010).

However, there was about $40 \%$ of their conceptual understanding in C3 concept has been completely transformed into alternative conception in two months later the learning program. This might explain why students' scores for conceptual understanding of light refraction decreased slightly from posttest to retention test. This result is consistent with She and Liao's (2010) study, which found that students' learning scores have decreased between posttest and retention tests. The argument is corresponding with the claims that students' level of pre-knowledge affected their learning outcomes from simulation (de Jong and van Joolingen 1998; Winberg and Berg 2007). In an addition, designing of the dual-situated learning events in this study may not be enough to provide specific mental sets that the students lack in the $\mathrm{C} 3$ concept and they need to use for fostering radical conceptual change on the concept.

\section{Conclusion}

This study demonstrates how instructions using simulation-based open inquiry with DSLM through these four dual-situational learning events helped students 
successfully construct scientific understanding of light refraction phenomena. The results suggested that incorporation of learning by simulation-based open inquiry into DSLM has the potential for the development of students' conceptual understanding in science through the mechanical process of conceptual change. Moreover, the change of their conception was a deep process of repairing students' alternative conceptions into scientific conception, called radical conceptual change, and the process of conceptual change could be fostered by the simulation-based open inquiry with DSLM. This implies that the simulation-based open inquiry with DSLM can be effective in fostering students' radical conceptual change in simulation-based open-inquiry learning environment as well as in web-based learning environment, or in the classroom. The results from this study could lead us to conclude that the simulation-based open-inquiry learning environment based on the DSLM could be an alternative way for developing conceptual understanding of light refraction and fostering the cognitive process of conceptual changes among students.

Acknowledgments This study is based on research financially supported by the Thailand Research Fund (TRF), the Commission on Higher Education (CHE), and Khon Kaen University (KKU). (Grant No. MRG5480058). An earlier version of this paper was presented at the 1st International Conference on Innovations in Education (ICIE 2012), Thailand; and the 3rd World Conference on Learning, Teaching and Educational Leadership (WCLTA 2012), Belgium.[Misc; Any opinions, findings, and conclusions or recommendations expressed in this materials are those of the authors and do not necessarily reflect the view of the TRF, CHE, and KKU.]

\section{References}

American Association for the Advancement of Science. (1993). Benchmarks for science literacy. New York: Oxford University Press.

Atasoy, Ş., \& Akdenız, A. R. (2007). Developing and applying a test related to appearing misconceptions about Newtonian laws of motion. Journal of Turkish Science Education, 4(1), 45-50.

Aydin, S., Keles, U. P., \& Hasiloglu, A. M. (2012). Establisment for misconceptions that science teacher candidates have about geometric optics. The online journal of new horizons in education, 2(3), 7-15.

Bell, R. L., \& Trundle, K. C. (2008). The use of a computer simulation to promote scientific conceptions of moon phases. Journal of Research in Science Teaching, 45(3), 346-372.

Berelson, B. (1952). Content analysis in communication research. New York: The Free Press.

Blake, C., \& Scanlon, E. (2007). Reconsidering simulations in science education at a distance: Features of effective use. Journal of Computer Assisted learning, 23(6), 491-502.

Buck, L. B., Bretz, S. L., \& Towns, M. H. (2008). Characterizing the level of inquiry in the undergraduate laboratory. Journal of College Science Teaching, 38(1), 52-58.

Carey, S. (1986). Cognitive science and science education. American Psychologist, 1, 1123-1130.

Chen, Y. L., Hong, Y. R., Sung, Y. T., \& Chang, K. E. (2011). Efficacy of simulation-based learning of electronics using visualization and manipulation. Educational Technology \& Society, 14(2), 269-277.

Chen, Y.-L., Pan, P.-R., Sung, Y.-T., \& Chang, K.-E. (2013). Correcting misconceptions on electronics: Effects of a simulation-based learning environment backed by a conceptual change model. Educational Technology \& Society, 16(2), 212-227.

Chiu, M. H., \& Lin, J. W. (2005). Promoting fourth graders' conceptual change of their understanding of electric current via multiple analogies. Journal of Research in Science Teaching, 42(4), 429-464.

Colella, V. (2000). Participatory simulation: Building collaborative understanding through immersive dynamic modeling. Journal of the Learning Sciences, 9(4), 471-500.

Cook, M. P. (2006). Visual representations in science education: The influence of prior knowledge and cognitive load theory on instructional design principles. Science Education, 90(6), 1073-1091. 
Cosgrove, M., \& Osborne, R. (1985). A teaching sequence on electric current. In R. Osborne \& P. Freyberg (Eds.), Learning in science: The implications of children's science. Auckland: Heinemann.

Cresswell, J. (2003). Research design: Qualitative, quantitative and mixed methods approaches. London: Sage.

de Jong, T., Linn, M. C., \& Zacharia, Z. C. (2013). Physical and virtual laboratories in science and engineering education. Science, 340, 305-308.

de Jong, T., Martin, E., Zamarro, J.-M., Esquembre, F., Swaak, J., \& van Joolingen, W. R. (1999). The integration of computer simulation and learning support; An example from the physics domain of collisions. Journal of Research in Science Teaching, 36, 597-615.

de Jong, T., \& van Joolingen, W. R. (1998). Scientific discovery learning with computer simulations of conceptual domains. Review of Educational Research, 68, 179-202.

Dega, B. G., Kriek, J., \& Mogese, T. F. (2013). Students' conceptual change in electricity and magnetism using simulations: A comparison of cognitive perturbation and cognitive conflict. Journal of Research in Science Teaching, 50(6), 677-698.

Dias, S. B., \& Diniz, J. A. (2014). Towards an enhanced learning management system for blended learning in higher education incorporating distinct learners' profiles. Educational Technology \& Society, 17, 307-319.

Djanett, B., Fouad, C., \& Djamel, K. (2013). What thinks the university's students about propagation of light in the vacuum? European Scientific Journal, 9(24), 197-213.

Duit, R., \& Treagust, D. (2003). Conceptual change: A powerful framework for improving science teaching and learning. International Journal of Science Education, 25, 671-688.

Dykstra, D. I., Boyle, C. F., \& Monarch, I. A. (1992). Studying conceptual change in learning physics. Science Education, 76, 615-652.

Fairclough, N. (2001). Critical discourse analysis as a method in social scientific research. In R. Wodak \& M. Meyer (Eds.), Methods of critical discourse analysis (pp. 121-138). London: Sage.

Fairclough, N. (2003). Analysing discourse: Textual analysis for social research. London/New York: Routledge.

Flick, L., \& Bell, R. (2000). Preparing tomorrow's science teachers to use technology: Guidelines for science educators. Contemporary Issues in Technology and Teacher Education [Online serial], 1(1). Retrieved from http://www.citejournal.org/vol1/iss1/currentissues/science/article1.htm.

Galili, I., \& Hazan, A. (2000). Learners' knowledge in optics: Interpretation, structure, and analysis. International Journal in Science Education, 22(1), 57-88.

Galili, I., \& Hazan, A. (2001). The effect of a history-based course in optics on students' views about science. Science \& Education, 10(1-2), 7-32.

Gerber, M., Grundt, S., \& Grote, G. (2008). Distributed collaboration activities in a blended learning scenario and the effects on learning performance. Journal of Computer Assisted learning, 24(3), 232-244.

Gunhaart, A., \& Srisawasdi, N. (2012). Effect of integrated computer-based laboratory environment on students' physics conceptual learning of sound wave properties. Procedia - Social and Behavioral Sciences, 46, 5750-5755.

Hake, R. R. (1998). Interactive-engagement versus traditional methods: A six-thousand student survey of mechanics test data for introductory physics courses. American Journal of Physics, 66(1), 64-74.

Hannon, J., \& Bretag, T. (2010). Negotiating contested discourses of learning technologies in higher education. Educational Technology \& Society, 13(1), 106-120.

Hanrahan, M. U. (2005). Highlighting hybridity: A critical discourse analysis of teacher talk in science classrooms. Science Education, 90(1), 8-43.

Hardy, C., Harley, B., \& Phillips, N. (2004). Discourse analysis and content analysis: Two solitudes? Qualitative Methods, 2, 19-22.

Hennessy, S., Deaney, R., \& Ruthven, K. (2006). Situated expertise in integrating use of multimedia simulation into secondary science teaching. International Journal of Science Education, 28(7), 701-732.

Hofstein, A., \& Lunetta, V. N. (2004). The laboratory in science education: Foundation for the 21st century. Science Education, 88, 28-54.

Hofstein, A., \& Mamlok-Naaman, R. (2007). The laboratory in science education: The state of the art. Chemistry Education: Research and Practice in Europe, 8(2), 105-108.

Hsu, Y. (2008). Learning about seasons in a technologically enhanced environment: The impact of teacher-guided and student-centered instructional approaches on the process of students' conceptual change. Science Education, 92, 320-344. 
Jaakkola, T., Nurmi, S., \& Veermans, K. (2011). A comparison of students' conceptual understanding of electric circuits in simulation only and simulation-laboratory contexts. Journal of Research in Science Teaching, 48, 71-93.

Jaakkolaa, T., \& Nurmi, S. (2008). Fostering elementary school students' understanding of simple electricity by combining simulation and laboratory activities. Journal of Computer Assisted learning, 24, 271-283.

Jimoyiannis, A., \& Angelaina, S. (2012). Towards an analysis framework for investigating students' engagement and learning in educational blogs. Journal of Computer Assisted learning, 28, 222-234.

Jimoyiannis, A., \& Komis, V. (2001). Computer simulations in teaching and learning physics: A case study concerning students' understanding of trajectory motion. Computers \& Education, 36(2), 183-204.

Kaewkhong, K., Mazzolini, A., Narumon Emarat, N., \& Arayathanitkul, K. (2010). Thai high-school students' misconceptions about and models of light refraction through a planar surface. Physics Education, 45(1), 91-107.

Kassarjian, H. H. (2001). Content analysis in consumer research. Journal of Consumer Research, 4, 8-18.

Koehler, M. J., Mishra, P., \& Yahya, K. (2007). Tracing the development of teacher knowledge in a design seminar: Integrating content, pedagogy and technology. Computers \& Education, 49(3), 740-762.

Krippendorff, K. (1980). Content analysis: An introduction to its methodology. Beverly Hills, CA: Sage.

Kuhn, D., Black, J. B., Kesselman, A., \& Kaplan, D. (2000). The development of cognitive skills to support inquiry learning. Cognition and Instruction, 18, 495-523.

Lazonder, A. W., \& Ehrenhard, S. (2013). Relative effectiveness of physical and virtual manipulatives for conceptual change in science: How falling objects fall. Journal of Computer Assisted learning,. doi:10.1111/jcal.12024.

Lazonder, A. W., Hagemans, M. G., \& de Jong, T. (2010). Offering and discovering domain information in simulation-based inquiry learning. Learning and Instruction, 20, 511-520.

Lee, C. Q., \& She, H. C. (2010). Facilitating students' conceptual change and scientific reasoning involving the unit of combustion. Research in Science Education, 40(4), 479-504.

Liao, Y. W., \& She, H. C. (2009). Enhancing eight grade students' scientific conceptual change and scientific reasoning through a web-based learning program. Educational Technology \& Society, 12(4), 228-240.

Lombardi, D., Sinatra, G. M., \& Nussbaum, E. M. (2013). Plausibility reappraisals and shifts in middle school students' climate change conceptions. Learning and Instruction, 27, 50-62.

Macabebe, E. Q. B., Culaba, I. B., \& Maquiling, J. T. (2010). Pre-conceptions of Newton's laws of motion of students in introductory physics. AIP Conference Proceedings, 1263(1), 106-109.

McElhaney, K. W., \& Linn, M. C. (2011). Investigations of a complex, realistic task: Intentional, unsystematic, and exhaustive experimenters. Journal of Research in Science Teaching, 48(7), 745-770.

Muller, D. A., Sharma, M. D., \& Reimann, P. (2008). Raising cognitive load with linear multimedia to promote conceptual change. Science Education, 92(2), 278-296.

National Research Council. (2000). How people learn: Brain, mind, experience, and school. Washington DC: National Academy Press.

Neuendorf, K. A. (2004). Content analysis-A contrast and complement to discourse analysis. Qualitative Methods, 2(1), 33-35.

Olympiou, G., \& Zacharia, Z. C. (2012). Blending physical and virtual manipulatives: An effort to improve students' conceptual understanding through science laboratory experimentation. Science Education, 96(1), 21-47.

Olympiou, G., Zacharia, Z. C., \& de Jong, T. (2013). Making the invisible visible: Enhancing students' conceptual understanding by introducing representations of abstract objects in a simulation. Instructional Science, 41, 575-596.

Phillips, N., \& Hardy, C. (2002). Discourse analysis: Investigating processes of social construction. Thousand Oaks, CA: Sage.

Posner, G. J., Strike, K. A., Hewson, P. W., \& Gertzog, W. A. (1982). Accommodation of a scientific conception: Toward a theory of conceptual change. Science Education, 66(2), 211-227.

Potter, J. (2003). Discourse analysis and discursive psychology. In P. M. Camic, J. E., Rhodes, \& L. Yardley (Eds.), Qualitative research in psychology: Expanding perspectives in methodology and design (pp. 73-94). Washington: American Psychological Association. 
Renken, M. D., \& Nunez, N. (2013). Computer simulations and clear observations do not guarantee conceptual understanding. Learning and Instruction, 23, 10-23.

Riffe, D., Lacy, S., \& Fico, F. G. (1998). Analyzing media messages: Using quantitative content analysis in research. Mahwah, NJ: Lawrence Erlbaum.

Ronen, M., \& Eliahu, M. (2000). Simulation-a bridge between theory and reality: The case of electric circuits. Journal of Computer Assisted learning, 16(1), 14-26.

Russ, R. S., Scherr, R. E., Hammer, D., \& Mikeksa, J. (2008). Recognizing mechanistic reasoning in student scientific inquiry: A framework for discourse analysis developed from philosophy of science. Science Education., 92(3), 499-525.

Rutten, N., van Joolingen, W. R., \& van der Veen, J. T. (2012). The learning effects of computer simulations in science education. Computers \& Education, 58(1), 136-153.

Saglam-Arslan, A., \& Devecioglu, Y. (2010). Student teachers' levels of understanding and model of understanding about Newton's laws of motion. Asia-Pacific Forum on Science Learning \& Teaching, 11(1), 1-20.

Savinainen, A., \& Scott, P. (2002). Using the Force Concept Inventory to monitor student learning and to plan teaching. Physics Education, 37, 53-58.

Schifter, C. C., Ketelhut, D. J., \& Nelson, B. C. (2012). Presence and middle school students' participation in a virtual game environment to assess science inquiry. Educational Technology \& Society, 15(1), 53-63.

She, H. C. (2002). Concepts of higher hierarchical level required more dual situational learning events for conceptual change: A study of students' conceptual changes on air pressure and buoyancy. International Journal of Science Education, 24(9), 981-996.

She, H. C. (2003). DSLM instructional approach to conceptual change involving thermal expansion. Research in Science and Technological Education, 21(1), 43-54.

She, H. C. (2004a). Facilitating changes in ninth grade students' understanding of dissolution and diffusion through DSLM instruction. Research in Science Education, 34(4), 503-525.

She, H. C. (2004b). Fostering radical conceptual change through dual-situated learning model. Journal of Research in Science Teaching, 41(2), 142-164.

She, H. C., \& Liao, Y. W. (2010). Bridging scientific reasoning and conceptual change through adaptive web-based learning. Journal of Research in Science Teaching, 47(1), 91-119.

Sing, C. C., \& Khine, M. S. (2006). An analysis of interaction and participation patterns in online community. Educational Technology \& Society, 9(1), 250-261.

Spyrtou, A., Hatzikraniotis, E., \& Kariotoglou, P. (2009). Educational software for improving learning aspects of Newton's Third Law for student teachers. Education and Information Technologies, 14(2), 163-187.

Srisawasdi, N. (2012). Introducing students to authentic inquiry investigation by using an artificial olfactory system. In K. C. D. Tan, M. Kim, \& S. W. Hwang (Eds.), Issues and challenges in science education research: Moving forward. Dordrecht: Springer.

Stewart, J., Cartier, J. L., \& Passmore, P. M. (2005). Developing understanding through model-based inquiry. In M. S. Donovan \& J. D. Bransford (Eds.), How students learn (pp. 515-565). Washington, DC: National Research Council.

Strike, K. A., \& Posner, G. J. (1985). A conceptual change view of learning and understanding. In L. West \& L. Pines (Eds.), Cognitive structure and conceptual change (pp. 211-231). Orlando. FL.: Academic Press.

Suits, J. P., \& Srisawasdi, N. (2013). Use of an interactive computer-simulated experiment to enhance students' mental models of hydrogen bonding phenomena. In J. P. Suits \& M. J. Sanger (Eds.), Pedagogic roles of animations and simulations in chemistry courses ACS Symposium Series 1142. Washington, DC: American Chemical Society.

Tao, P. K., \& Gunstone, R. F. (1999). The process of conceptual change in force and motion during computer-supported physics instruction. Journal of Research in Science Teaching, 36, 859-882.

Thagard, P. (1992). Conceptual revolutions. Princeton, NJ: Princeton University Press.

Trundle, K. C., \& Bell, R. L. (2010). The use of a computer simulation to promote conceptual change: A quasi-experimental study. Computers \& Education, 54, 1078-1088.

Tseng, C. H., Tuan, H. L., \& Chin, C. C. (2009). Investigating the influence of motivational factors on conceptual change in a digital learning context using the dual-situated learning model. International Journal of Science Education, 32(14), 1853-1875. 
Veemans, K., van Joolingen, W., \& de Jong, T. (2006). Use of heuristics to facilitate scientific discovery learning in a simulation learning environment in a physics domain. International Journal of Science Education, 28(4), 341-361.

Vreman-de Olde, C., de Jong, T., \& Gijlers, H. (2013). Learning by designing instruction in the context of simulation-based inquiry learning. Educational Technology \& Society, 16(4), 47-58.

Weber, R. P. (1985). Basic content analysis. Beverly Hills, CA: Sage.

Wilson, J. (2003). Political discourse. In D. Schffrin, et al. (Eds.), The handbook of discourse analysis (pp. 398-415). Malden: Blackwell Publishing Company.

Winberg, T. M., \& Berg, C. A. R. (2007). Students' cognitive focus during a chemistry laboratory exercise: Effects of a computer-simulated prelab. Journal of Research in Science Teaching, 44(8), 1108-1133.

Windschitl, M., \& Andre, T. (1998). Using computer simulations to enhance conceptual change: The roles of constructivist instruction and student epistemological beliefs. Journal of Research in Science Teaching, 35(2), 145-160.

Wu, H.-K., \& Shah, P. (2004). Thinking with representations: Exploring visuospatial thinking in chemistry. Science Education, 88(3), 465-492.

Yen, H. C., Tuan, H. L., \& Liao, C. H. (2011). Investigating the influence of motivation on students' conceptual learning outcomes in web-based vs. classroom-based science teaching contexts. Research in Science Education, 41, 211-224.

Zacharia, Z. C. (2007). Comparing and combining real and virtual experimentation: An effort to enhance students' conceptual understanding of electric circuits. Journal of Computer Assisted learning, 23, $120-132$.

Zacharia, Z., \& Anderson, O. (2003). The effects of an interactive computer-based simulation prior to performing a laboratory inquiry-based experiment on students' conceptual understanding of physics. American Journal of Physics, 71, 618.

Zacharias, G. L., MacMillan, J., \& Van Hemel, S. B. (2008). Behavioral modeling and simulation: From individuals to societies. Washington, DC: National Academies Press.

Zion, M., \& Sadeh, I. (2007). Curiosity and open inquiry learning. Journal of Biological Education, 41(4), 162-168. 